\title{
Making brundlefly, one gene at a time
}

\author{
Xiaomeng Zhang ${ }^{1,3}$, Helena E Richardson ${ }^{2,4}$, Kieran F Harvey ${ }^{1,3}$ \\ ${ }^{1}$ Cell Growth and Proliferation Laboratory, and ${ }^{2}$ Cell Cycle and Development Laboratory, Peter MacCallum Cancer Centre, 7 St \\ Andrews Place, East Melbourne, Victoria, Australia, 3002; ${ }^{3}$ Department of Pathology, and ${ }^{4}$ Department of Anatomy and Cell Biology, \\ University of Melbourne, Parkville, Victoria, Australia, 3010
}

Cell Research (2009) 19:5-7. doi: 10.1038/cr.2009.2; published online 5 January 2009

Drosophila melanogaster has proved to be a remarkable organism for studying complex biological phenomenona. One of the great virtues of $D$. melanogaster is its amenability to large scale genetic screens. Many types of genetic screen have been performed in D. melanogaster in the past with great success, to explore myriad biological processes such as tissue growth, differentiation, patterning, morphogenesis, and immunity. Different types of genetic screens employed include: (1) Homozygous mutant. A striking example of which is the embryonic patterning screen performed by Wieschaus and Nusslein-Volhard, where multiple genes important for the development and patterning of the $D$. melanogaster embryo were identified [1]. (2) Dominant modifier. These have most commonly been used to identify new components of existing signalling pathways. A highly successful example was the identification of components of the Ras-Map kinase signalling pathway as downstream effectors of the Sevenless receptor tyrosine kinase [2]. (3) Overexpression. These screens typically employ a collection of randomly inserted transposable elements that activate the expression of different genes when crossed to tissue-specific drivers [3]. (4) Flp/FRT clonal. Homozygous

Correspondence: Kieran F Harvey

Tel: 6139656 1291; Fax: 61396561411

E-mail: kieran.harvey@petermac.org mutations in many genes cause lethality at early stages of development. A major advantage of clonal screens is that they provide a mechanism to generate patches of homozygous mutant tissue in an otherwise heterozygous animal, thus allowing phenotypic characterisation of lethal genes at later stages of development [3]. (5) RNA interference. First performed in cell culture, and more recently in transgenic D. melanogaster, these screens utilise the phenomenon of RNA interference to systematically ablate the function of individual genes in a given experimental setting. Cell culture screens have largely focussed on identification of novel modulators of existing signalling pathways $[4,5]$.

In the November issue of Cell Research, Tian $\mathrm{Xu}$ and colleagues [6] describe a genetic overexpression screen whereby they aim to classify the function of uncharacterised human genes by overexpressing them in $D$. melanogaster. The screen was conceived based on the premise that most important biological processes are conserved between human and flies, and that although the identity of all human genes is now known, the function of many is still obscure [7-9].

$\mathrm{Xu}$ et al. [6] generated transgenic flies expressing 236 different human cDNAs under the control of the yeast GAL4 UAS control element, allowing expression of these upon crossing the flies to tissue-specific GAL4 drivers. They used drivers that expressed ubiq- uitously, in the developing eye or wing epithelia, or in differentiating neural cells, and screened for cDNAs that when overexpressed, produced visible phenotypes. They found that 51 of the human cDNAs analysed in flies gave a reproducible phenotype. Through comparing the phenotypes generated to those obtained in other studies, by searching the vast $D$. melanogaster literature (see http://flybase.bio.indiana. edu/), it can be inferred as to whether the over-expression of the gene affects processes such as cell growth, proliferation, death, differentiation or morphology. Moreover, the phenotype generated by over-expression of the human cDNA can then be interrogated against a bank of available $D$. melanogaster mutations to provide insight into the pathway by which the gene operates. This approach can be scaled-up to a global context to analyse all $\sim 30000$ human cDNAs in D. melanogaster, and since the function of at least $40 \%$ of all predicted human coding cDNAs is not yet known, this strategy has the potential to rapidly provide valuable information on the function of "pioneer" human genes.

While conceptually this approach has immense power in revealing the function of unknown human genes, there are many caveats to the success of this approach and whether the information revealed in the $D$. melanogaster system will be meaningful for mammalian biology. Firstly, it is likely that only highly conserved genes will give phenotypes 
when mis-expressed in a heterologous system (e.g. Rpl8 which is $80 \%$ identical between human and D. melanogaster). Secondly, even amongst the most highly conserved genes, only those that are rate-limiting for a particular process in D. melanogaster may be expected to give phenotypes, rather knockdown of gene-function is more likely to create phenotypes. In this regard, it is pertinent to point out that one of the cDNAs (the ribosomal protein gene, $R P L 8$ ) that $\mathrm{Xu}$ et $a l$., discovered to produce strong phenotypes when expressed from all drivers was shown to be a truncated dominant-negatively acting protein. Thirdly, due to divergence of critical domains in homologous proteins between D. melanogaster and humans, and differences in protein complexes or signalling pathways between organisms, many human protein functions may be missed. Moreover, neomorphic effects may occur when the human protein is expressed in D. melanogaster that do not reflect the physiological function of the human protein. This issue is the most problematic in that it may lead to misconceptions in the understanding of gene function, and therefore it is highly important that all phenotypic effects are validated in the $D$. melanogaster system, as well as in mammalian cells (see Figure 1).

Importantly, Xu et al. provide convincing data to support the validity of their screening approach. They focus on three human proteins that give phenotypes upon expression from various drivers; the cell cycle inhibitor, p27, the SFRS3 splicing factor, and the ribosomal protein L8. p27-Kip1 is a homolog of the D. melanogaster protein Dacapo, which binds to and inactivates

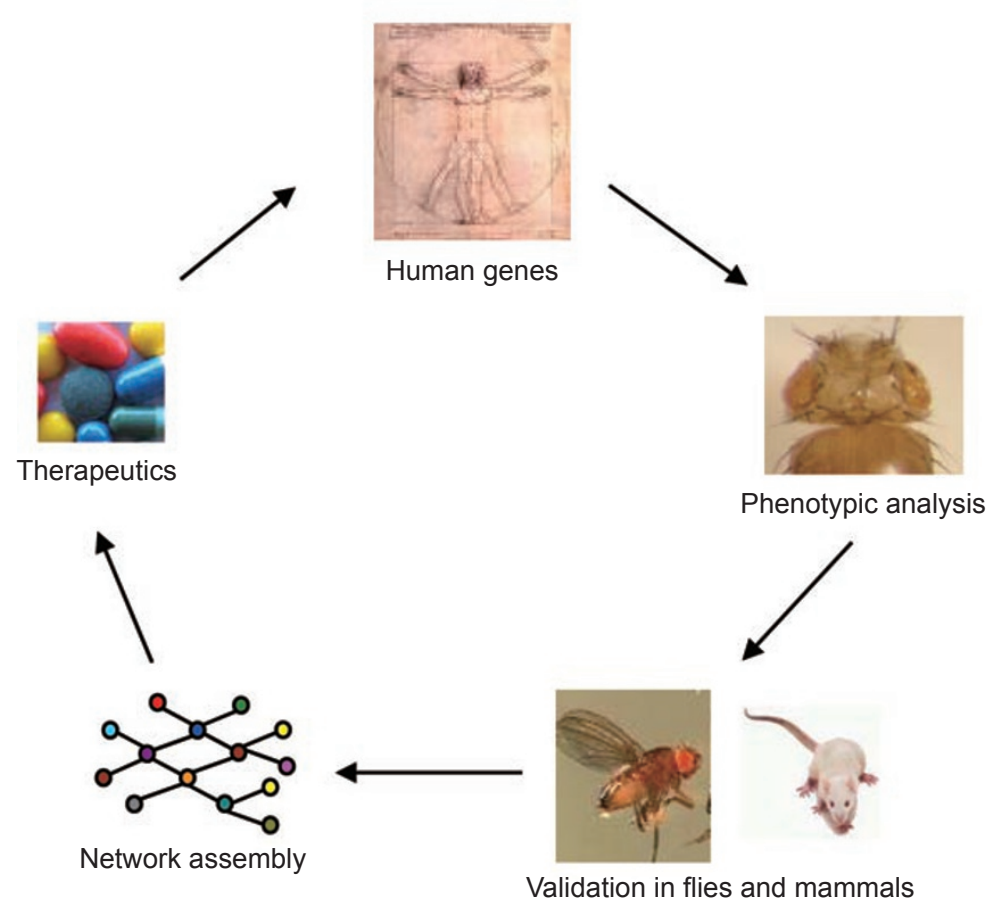

Figure 1 Theme for discovery of human gene function in $D$. melanogaster. Human genes are mis-expressed in different $D$. melanogaster tissues and the resultant phenotypes scored. Results are then studied in detail by assessing the function of phenotype-eliciting genes and their $D$. melanogaster orthologues (if available) in mammalian tissue culture, flies and mice. Signalling networks are assembled by grouping known and novel genes with related phenotypes. Suitable therapeutic targets are selected for the appropriate disease, and drugs developed.

G1-S phase Cyclin-dependent protein kinases, resulting in cell cycle arrest at the G1-S phase transition. Consistent with this, overexpression of $\mathrm{p} 27$ in the developing eye (via eyeless-GAL4) results in a small eye, while expression in the differentiating cells of the eye (via GMR-GAL4) results in a rough eye phenotype. Although they did not examine the effect of p27 expression on cell cycle progression in this study, these phenotypes are similar to that obtained when Dacapo or another human homolog of Dacapo, p21-Cip1 are overexpressed in the D. melanogaster eye, which have been shown to block S phase entry [10]. Moreover, they show the importance of a critical phosphorylation site on p27, Serine 10, which is important to allow nuclear entry and stability of p27, and for its activity in human cells and $D$. melanogaster tissues. This result demonstrates the functional conservation of Serine 10 phosphorylation for p27 function in D. melanogaster cells, and suggests that this system will be useful for further analyses to investigate $\mathrm{p} 27$ regulation.

$\mathrm{Xu}$ et al., further demonstrate the validity of their approach to investigate human gene function in D. melanogaster, from their analysis of the splicing factor gene, SFRS3, and the gene encoding the ribosomal protein, RPL8. Expression of $S F R F 3$ in the differentiating region of the eye (via GMR-GAL4) results in a rough eye phenotype, while expression in the developing genital discs (via Patched-GAL4) leads to intersex genitalia. Importantly, overexpression of the D. melanogaster homolog of SFRS3, Dx16, in these tissues results in identical phenotypes. A dominant negative version of RPL8 reduced tissue size and could be rescued by expression of $D$. melanogaster RPL8. In addition, Xu et al., highlighted the functional conservation between human and D. melanogaster RPL8 by showing that co-expression of either D. melanogaster $R P L 8^{R N A i}$ or human $R P L 8^{D N}$ could reverse overgrowth as- 
sociated with hyperactivation of the insulin, or target of rapamycin pathways, which are known to stimulate ribosome biogenesis. These studies show that the expression of the human orthologues of SFRS3 and RPL8 are likely to be working by a similar manner to their $D$. melanogaster counterparts, and support the use of the D. melanogaster system to investigate the mechanism of action of SFRS3 and RPL8.

Currently the $\mathrm{Xu}$ laboratory has published a screen covering $1 \%$ of the human genome for overexpression phenotypes in D. melanogaster. An enormous expansion in effort and resources will be required to tackle the ambitious task of extending this approach to the entire human genome. Such a task is not insurmountable however, but will require a highly efficient approach. Recently the Vienna Drosophila Research Center, led by Barry Dickson, generated approximately 20000 transgenic D. melanogaster lines expressing RNA interference constructs [5]. Approximately 30000 transgenic overexpression lines would need to be produced to cover the human genome (assuming one transgenic fly line per gene). Based on the current study by $\mathrm{Xu}$ et al., more than 1500 transgenic fly lines were generated for 236 genes, (roughly 6 transgenic fly lines per gene), meaning that approximately 200000 transgenic fly lines would need to be produced to cover the whole human genome. To increase the efficiency of a genome-wide study it would be wise to employ recent technological advances in the production and expression of transgenes in D. melanogaster, such as site-specific integration, and transgenic vectors with gene insulator elements that boost expression from heterologous promoters [11]. Theoretically the utilisation of such technologies would reduce the time taken to generate transgenic D. melanogaster, guarantee robust and consistent transgene expression, and ensure that only one transgene would need to be generated for each human gene. If this approach of humanising the fly, one-gene-at-a-time, can be achieved, it has enormous potential for the discovery of new functions for human genes, and moreover, this bank of transgenic flies could be used in a "new-age" genetic interaction screen to discover novel human gene networks (see Figure 1).

\section{References}

1 Nusslein-Volhard C, Wieschaus E. Mutations affecting segment number and polarity in Drosophila. Nature 1980; 287:795-801.

2 Simon MA, Bowtell DD, Dodson GS, Laverty TR, Rubin GM. Ras1 and a putative guanine nucleotide exchange factor perform crucial steps in signaling by the sevenless protein tyrosine kinase.
Cell 1991; 67:701-716.

3 Blair SS. Genetic mosaic techniques for studying Drosophila development. Development 2003; 130:5065-5072.

4 Perrimon N, Mathey-Prevot B. Applications of high-throughput RNA interference screens to problems in cell and developmental biology. Genetics 2007; 175:7-16.

5 Dietzl G, Chen D, Schnorrer F, et al. A genome-wide transgenic RNAi library for conditional gene inactivation in Drosophila. Nature 2007; 448:151-156.

$6 \mathrm{Xu} \mathrm{R}$, Deng K, Zhu Y, et al. A large-scale functional approach to uncover human genes and pathways in Drosophila. Cell Res 2008; 18:1114-1127.

7 Harvey K, Tapon N. The SalvadorWarts-Hippo pathway - an emerging tumour-suppressor network. Nat Rev Cancer 2007; 7:182-191.

8 Brumby AM, Richardson HE. Using Drosophila melanogaster to map human cancer pathways. Nat Rev Cancer 2005; 5:626-639.

9 Fortini ME, Skupski MP, Boguski MS, Hariharan IK. A survey of human disease gene counterparts in the Drosophila genome. J Cell Biol 2000; 150:F23F30.

10 de Nooij JC, Hariharan IK. Uncoupling cell fate determination from patterned cell division in the Drosophila eye. Science 1995; 270:983-985.

11 Markstein M, Pitsouli C, Villalta C, Celniker SE, Perrimon N. Exploiting position effects and the gypsy retrovirus insulator to engineer precisely expressed transgenes. Nat Genet 2008; 40:476483. 